\title{
УСТОЙЧИВОСТЬ ТОЧКИ РЕШЕНИЯ НЕЛИНЕЙНОГО УРАВНЕНИЯ, ЗАВИСЯЩЕГО ОТ ПАРАМЕТРА
}

\author{
(Представил Н. Алумяэ)
}

В работе рассматривается нелинейное уравнение в предположении, что его решение при некотором фиксированном значении параметра, от которого оно зависит, известно. Указываются условия, гарантирующие существование решения уравнения и при некоторых других значениях параметра и малое изменение точки решения, если параметр мало изменяется. Результаты применяются к изучению уравнения со случайным параметром.

1. Пусть задано зависящее от параметра a семейство отображеннй $F(\cdot, a): D(a) \rightarrow R^{n}, D(a) \subset R^{n}$, где значения $a$ принадлежат некоторому множеству $A, A \subset R^{s}$. Предположим, что для каждого значения параметра $a \in A$ отображение $F(x, a)$ непрерывно дифференцируемо на $D(a)$.

Пусть известно, что уравнение

$$
F\left(x, a_{0}\right)=0,
$$

где $a_{0} \in A$, имеет решение $x\left(a_{0}\right)$. Будем искать условия, при которых уравнение

$$
F(x, a)=0
$$

имеет решение $x(a)$, если $а$ достаточно близок к $a_{0}$, и оценим расстояние между $x(a)$ и $x\left(a_{0}\right)$. При этом нам понадобится

Т еорем а $1\left[{ }^{1}\right]$. Пусть $f: Q \rightarrow R^{n}, Q \subset R^{n}$, непрерывно дифференцируема на $Q$ и пусть имеется такой открытый шар $S\left(x_{0}, r\right) \subset Q$, $S\left(x_{0}, r\right)=\left\{x \mid\left\|x-x_{0}\right\|<r\right\}$, что $\left\|f^{\prime}(x)^{-1}\right\|<\gamma \quad$ при $\quad x \in S\left(x_{0}, r\right) \quad$ u $r .>\gamma\left\|f\left(x_{0}\right)\right\|$. Тогда уравнение $f(x)=0$ имеет решение в $S\left(x_{0}, r\right)$.

Связь между уравнениями (1) и (2) доказывает

Т еорем а 2. Пусть выполнены условия

1. $\left\|F^{\prime}{ }_{x}\left(x_{1}, a_{0}\right)^{-1}-F^{\prime}{ }_{x}\left(x_{2}, a_{0}\right)^{-1}\right\| \leqslant C_{1}\left\|x_{1}-x_{2}\right\|$ для всех $x_{1}, x_{2} \in R^{n}$.

2. $\left\|F\left(x\left(a_{0}\right), a_{1}\right)-F\left(x\left(a_{0}\right), a_{2}\right)\right\| \leqslant C_{2}\left\|a_{1}-a_{2}\right\|$ для всех $a_{1}, a_{2} \in A$.

3. $\left\|F^{\prime}{ }_{x}\left(x, a_{1}\right)^{-1}-F^{\prime}{ }_{x}\left(x, a_{2}\right)^{-1}\right\| \leqslant C_{3}\left\|a_{1}-a_{2}\right\|$ для всех $x \in R^{n} u a_{1}, a_{2} \in A$.

Тогда,

1) если а фиксирован, $0<\left\|a-a_{0}\right\|<1 / C_{1} C_{2}$, то уравнение (2) имеет решение $x(a)$ в шаре $S\left(x\left(a_{0}\right), \varepsilon\right)$,

$$
\varepsilon>C_{2}\left\|a-a_{0}\right\|\left(C_{3}\left\|a-a_{0}\right\|+\left\|F^{\prime}{ }_{x}\left(x\left(a_{0}\right), a_{0}\right)^{-1}\right\|\right) /\left(1-C_{1} C_{2}\left\|a-a_{0}\right\|\right),
$$

2) если в фиксировано, то (2) имеет решение $x(a)$ в шаре $S\left(x\left(a_{0}\right), \varepsilon\right)$ при всех значениях параметра а, удовлетворяющих

$$
\left\|a-a_{0}\right\|<\left(\sqrt{\gamma^{2}(\varepsilon)+4 C_{3} \varepsilon / C_{2}}-\gamma(\varepsilon)\right) / 2 C_{3} \text { в случае } C_{3} \neq 0
$$




$$
\left\|a-a_{0}\right\|<\varepsilon / C_{2} \gamma(\varepsilon) \text { в случае } C_{3}=0,
$$

где $\gamma(\varepsilon)=\sup _{x \in S\left(x\left(a_{0}\right), \varepsilon\right)}\left\|F_{x}^{\prime}(x, \varepsilon)^{-1}\right\|$.

Доказательство. Пусть параметр а фиксирован. Для того чтобы уравнение (2) имело решение, достаточно потребовать выполнимости условий теоремы 1. Очевидно, если существует $\varepsilon>0$ такое, что

$$
\varepsilon>\left[\sup _{x \in S\left(x\left(a_{0}\right), \varepsilon\right)}\left\|F^{\prime}{ }_{x}(x, a)^{-1}\right\|\right]\left\|F\left(x\left(a_{0}\right), a\right)\right\|,
$$

то уравнение (2) имеет решение в шаре $S\left(x\left(a_{0}\right), \varepsilon\right)$. В силу условия 3 имеем $\quad\left|\left\|F^{\prime}{ }_{x}(x, a)^{-1}\right\|-\left\|F^{\prime}{ }_{x}\left(x, a_{0}\right)^{-1}\right\|\right| \leqslant\left\|F_{x}^{\prime}(x, a)^{-1}-F_{x}^{\prime}\left(x, a_{0}\right)^{-1}\right\| \leqslant$ $\leqslant C_{3}\left\|a-a_{0}\right\|, \quad$ откуда $\quad\left\|F^{\prime}{ }_{x}(x, a)^{-1}\right\| \leqslant\left\|F^{\prime}{ }_{x}\left(x, a_{0}\right)^{-1}\right\|+C_{3}\left\|a-a_{0}\right\|$ и sup $\left\|F^{\prime}{ }_{x}(x, a)^{-1}\right\| \leqslant \sup _{x \in S\left(x\left(\frac{1}{2}\right)\right.}\left\|F^{\prime}{ }_{x}\left(x, a_{0}\right)^{-1}\right\|+C_{3}\left\|a-a_{0}\right\| . \quad$ Из усло$x \in S\left(x\left(a_{0}\right), \varepsilon\right) \quad x \in S\left(x\left(a_{0}\right), \varepsilon\right)$

вия 2 легко получается $\left\|F\left(x\left(a_{0}\right), a\right)\right\|=\left\|F\left(x\left(a_{0}\right), a\right)-F\left(x\left(a_{0}\right), a_{0}\right)\right\| \leqslant$ $\leqslant C_{2}\left\|a-a_{0}\right\|$. Следовательно, если $\varepsilon$ удовлетворяет неравенству

$$
\varepsilon>C_{2}\left\|a-a_{0}\right\|\left(C_{3}\left\|a-a_{0}\right\|+\sup _{x \in \mathrm{S}\left(x\left(a_{0}\right), \varepsilon\right)}\left\|F^{\prime}{ }_{x}\left(x, a_{0}\right)^{-1}\right\|\right),
$$

то оно удовлетворяет и неравенству (6). Далее, в силу условия 1 $\left\|F^{\prime}{ }_{x}\left(x, a_{0}\right)^{-1}-F_{x}^{\prime}\left(x\left(a_{0}\right), a_{0}\right)^{-1}\right\| \leqslant C_{1}\left\|x-x\left(a_{0}\right)\right\| \leqslant C_{1} \varepsilon$ при $x \in S\left(x\left(a_{0}\right), \varepsilon\right)$, откуда $\left\|F^{\prime}{ }_{x}\left(x, a_{0}\right)^{-1}\right\| \leqslant\left\|F^{\prime}{ }_{x}\left(x\left(a_{0}\right), a_{0}\right)^{-1}\right\|+C_{1} \varepsilon$ и $\sup _{x \in S\left(x\left(a_{0}\right), \varepsilon\right)}\left\|F^{\prime}{ }_{x}\left(x, a_{0}\right)^{-1}\right\| \leqslant$ $\leqslant\left\|F^{\prime}{ }_{x}\left(x\left(a_{0}\right), a_{0}\right)_{0}^{-1}\right\|+C_{1} \varepsilon$. Аналогично, если $\varepsilon$ удовлетворяет неравенству

$$
\varepsilon>C_{2}\left\|a-a_{0}\right\|\left(C_{3}\left\|a-a_{0}\right\|+\left\|F_{x}^{\prime}\left(x\left(a_{0}\right), a_{0}\right)^{-1}\right\|+C_{1} \varepsilon\right),
$$

то оно удовлетворяет и (7), а следовательно, и (6). Выразим из (8) $\varepsilon$, используя предположение $\left\|a-a_{0}\right\|<1 / C_{1} C_{2}$. Получим $\varepsilon>C_{2}\left\|a-a_{0}\right\| X$ $\times\left(C_{3}\left\|a-a_{0}\right\|+\left\|F^{\prime}{ }_{x}\left(x\left(a_{0}\right), a_{0}\right)^{-1}\right\|\right) /\left(1-C_{1} C_{2}\left\|a-a_{0}\right\|\right)$.

Во втором случае, когда $\varepsilon$ фиксировано, будем исходить из неравенства (7). Если $C_{3}=0$, то с учетом обозначений теоремы оценку (5) получим непосредственно. Если $C_{3} \neq 0$, то $(7)$ является квадратичным относительно $\left\|a-a_{0}\right\|$ неравенством, и оно выполняется, если $\left\|a-a_{0}\right\|$ удовлетворяет (4). Теорема доказана.

3 а меч ан ие. Теорема 2 точна в определенном смысле для линейного уравнения

$$
B x=a,
$$

где $B$ - симметричная матрица с полным рангом. Действительно, в данном случае $C_{1}=C_{3}=0, C_{2}=1, \gamma(\varepsilon)=\gamma=\left\|B^{-1}\right\|$. Решение уравнения (9) $-x(a)=B^{-1} a$. Если $a-$ собственный вектор матрицы $B^{-1}$, соответствующий наибольшему по абсолютной величине собственному значению, то $\|x(a)\|=\left\|B^{-1}\right\|\|a\|$. В то же время по оценке (3) $x(a)$ находится в шаре $S(0, \varepsilon), \varepsilon,>\left\|B^{-1}\right\|\|a\|$. Следовательно, при выбранном $a$ неравенство (3) для уравнения (9) не улучшаемо. Неулучшаемость неравенства (5) для рассматриваемого случая показывается аналогичным образом.

2. Перейдем теперь к изучению уравнения со случайным параметром

$$
F(x, \xi(\omega))=0,
$$

где $\xi(\omega)=\left(\xi_{1}(\omega), \xi_{2}(\omega), \ldots, \xi_{s}(\omega)\right)$ - измеримая вектор-функция из $\Omega$ в $R^{s}$. Пусть $E$ обозначает математическое ожидание и $\sigma$ дисперсию. Предположим, что нелинейное уравнение

$$
F(x, E \xi(\omega))=0
$$


имеет решение $x(E \xi(\omega))$. Обозначим $\Gamma(\omega)=\{x \mid F(x, \xi(\omega))=0\}$. На основе теоремы 2 легко показать существование измеримого множества $\Omega^{\prime}, \Omega^{\prime} \subset\{\omega \mid \Gamma(\omega) \neq \varnothing\}$. В самом деле, в случае выполнимости условий $1-3$, если только $\|\xi(\omega)-E \xi(\omega)\|<1 / C_{1} C_{2}$, уравнение (10) имеет решение $x(\xi(\omega))$. Очевидно, что множество $\Omega^{\prime}=\{\omega \mid \| \xi(\omega)-$ $\left.-E \xi(\omega) \|<1 / C_{1} C_{2}\right\}$ измеримо и содержится во множестве $\{\omega \mid \Gamma(\omega) \neq \varnothing\}$. Таким образом, вероятность события, что (10) разрешимо, можно оценить с помощью неравенства типа Чебышева $\left[{ }^{2}\right]$, откуда получим

$$
P\left(\Omega^{\prime}\right)=P\left\{\omega \mid\|\xi(\omega)-E \xi(\omega)\|<1 / C_{1} C_{2}\right\} \geqslant 1-C_{1}^{2} C_{2}^{2} \sum_{i=1}^{s} \sigma^{2} \xi_{i}(\omega) .
$$

Приведем вероятностные оценки расстояний между решениями уравнений (10) и (11). Если $C_{3} \neq 0$, то с учетом (4) имеем

$$
\begin{gathered}
P\{\omega \mid\|x(\xi(\omega))-x(E \xi(\omega))\|<\varepsilon\} \geqslant \\
\geqslant P\left\{\omega \mid\|\xi(\omega)-E \xi(\omega)\|<\left(\sqrt{\gamma^{2}(\varepsilon)+4 C_{3} \varepsilon / C_{2}}-\gamma(\varepsilon)\right) / 2 C_{3}\right\} \geqslant \\
\geqslant 1-2 C_{3}^{2} \sum_{i=1}^{s} \sigma^{2}(\xi i(\omega)) /\left(2 C_{3} \varepsilon / C_{2}-\gamma(\varepsilon) \sqrt{\gamma^{2}(\varepsilon)+4 C_{3} \varepsilon / C_{2}}\right),
\end{gathered}
$$

а если $C_{3}=0$, то с учетом (5) имеем

$$
P\{\omega \mid\|x(\xi(\omega))-x(E \xi(\omega))\|<\varepsilon\} \geqslant 1-C_{2}^{2} \gamma^{2}(\varepsilon) \sum_{i=1}^{8} \sigma^{2}\left(\xi_{i}(\omega)\right) / \varepsilon^{2} .
$$

Наконец, докажем теорему об измеримости $x(\xi(\omega))$, т. е. приведем условия, при которых (10) имеет случайное решение. Напомним, что точечно-множественным отображением $\Gamma$ из множества $X$ во множество $Y$ называется отображение, которое каждому элементу $x \in X$ ставит в соответствие некоторое множество из $Y\left[{ }^{3}\right]$. Если на $X$ задана мера, а $Y$ является топологическим пространством, то $\Gamma$ называется измеримым, если множество $\Gamma^{-} \mathbb{W}=\{x \in X \mid \Gamma(x) \cap W \neq \varnothing\}$ измеримо при каждом закрытом множестве $W \subset Y\left[{ }^{3}\right]$. Измеримое точечно-множественноє отображение из вероятностного пространства в пространство $Y$ называется случайным подмножеством пространства $Y$ [ [ $\left.{ }^{4}\right]$. По аналогии с определением случайной неподвижной точки случайного уравнения [ $\left.{ }^{4}\right]$ будем называть случайным решением уравнения (10) на случайном подмножестве $U$ пространства $R^{n}$ измеримое отображение $\eta(\omega)$ из $\Omega$ в $R^{n}$, если для почти всех $\omega$ выполняются два условия: 1) $\eta$ является селектором случайного подмножества $U$, т. е. $\eta(\omega) \in U(\omega)$, и 2) $F(\eta(\omega), \xi(\omega)) '=0$.

Т ео рем а 3 . Пусть функция $F(x, a)$.епрерывна по а и пусть, кроме того, выполнены условия теоремь 2. Пусть уравнение (11) имеет решение $x(E \xi(\omega))$. Если $\|\xi(\omega)-E \xi(\omega)\|<1 / C_{1} C_{2}$ для почти всех $\omega$, тогда уравнение (10) имеет случайное решение, которое является измеримым селектором случайного шара

2วe

$$
S=S(x(E \xi(\omega)), d(\|\xi(\omega)-E \xi(\omega)\|)+\gamma),
$$

$$
\begin{gathered}
d(\|\xi(\omega)-E \xi(\omega)\|)=C_{1}\|\xi(\omega)-E \xi(\omega)\| \times \\
X\left(C_{3}\|\xi(\omega)-E \xi(\omega)\|+\left\|F^{\prime}{ }_{x}(x(E \xi(\omega)), E \xi(\omega))^{-1}\right\|\right) \\
/\left(1-C_{1} C_{2}\|\xi(\omega)-E \xi(\omega)\|\right)
\end{gathered}
$$

при любом фиксированном $\gamma>0$. 
Дока з а тельст в о. Если $\omega \in \Omega^{\prime}, \Omega^{\prime}=\{\omega \in \Omega \mid\|\xi(\omega)-E \xi(\omega)\|<$ $\left.<1 / \dot{C}_{1} C_{2}\right\}$, то по доказанному выше уравнение (10) имеет решение в шаре $S=S(x(E \xi(\omega)), d(\|\xi(\omega)-E \xi(\omega)\|)+\gamma)$. Другими словами, $\Gamma(\omega) \neq \varnothing$ для всех $\omega \in \Omega^{\prime}$. Кроме того, очевидно, что $\Gamma(\omega)$ замкнуто. Далее, при любом замкнутом $W, \Gamma(\omega) \cap W=\varnothing$, из-за непрерывности $F(x, a)$ по $x$ имеем $\{\omega \mid \Gamma(\omega) \cap W \neq \varnothing\}=\{\omega \mid F(x, \xi(\omega))=0$ для некоторого $x \in W\}=\bigcap_{n=1}^{\infty} \bigcup_{i=1}^{\infty}\left\{\omega \mid\left\|F\left(x_{i}, \xi(\omega)\right)\right\|<1 / n\right\}$, где $x_{i}$ образуют счетное всюду плотное в $W$ множество (напр., множество векторов, координаты которых - рациональные числа). В силу предположения о непрерывности функции $F(x, a)$ по $a$, множество $\left\{\omega \mid\left\|F\left(x_{i}, \xi(\omega)\right)\right\|<1 / n\right\}$ измеримо при любом $i$, а также измеримы сумма и объединение счетного числа измеримых множеств. Следовательно, точечно-множественное отображение $\Gamma: \Omega^{\prime} \rightarrow R^{n}$ измеримо по определению и по [ $\left.{ }^{3}\right]$ имеет измеримый селектор.

\section{Л И Т Е Р А Т Р А}

1. Ортега Дж., Рейнбольдт В., Итерационные методы решения нелинейных систем уравнений со многими неизвестными, М., «Мир», 1975.

2. T a m m, E., Math. Operationsforsch. Statist., Ser. Optimization, 11, № 3, 487-497 $(1980)$.

3., C a st a i g n, Ch., Rev. Franç. Inform. et Rech. Opér., № 1, 91-126 (1967).

4. B o c\$a n, Gh., Rev. Roum. Math. Pures Appl., 26, № 3, 375-379 (1981).

Ннститут кибернетики

Академии наук Эстонской ССР
Поступила в редакцию 27 апреля 1982

Ebu TAMM

\section{PARAMEETRIST SOLTUVA MITTELINEAARSE VORRANDI LAHENDI STABIILSUS}

Töös on uuritud parameetrist sōltuva mittelineaarse võrrandi lahenduvust ja lahendi asukohta eukleidilises ruumis $R^{n}$. Eeldusel, et parameetri mingi fikseeritud väärtuse korral on vaadeldav võrrand lahenduv ja lahend teada, on näidatud parameetri võimalikku muutumist tema antud väärtuse ümber nii, et võrrand jääb lahenduvaks. On hinnatud ka vastava lahendi kaugust teadaolevast lahendist.

Tulemuste põhjal on hinnatud juhuslikust parameetrist sõltuva võrrandi lahenduvuse tõenäosust ja lahendi kaugust niisuguse determineeritud vōrrandi lahendist, kus juhuslik parameeter on asendatud tema matemaatilise ootusega. Lõpuks on tõestatud teoreem juhuslikust parameetrist sōltuva võrrandi juhusliku lahendi olemasolu kohta.

\section{Ebu TAMM}

\section{STABILITY OF A SOLUTION OF A NONLINEAR EQUATION DEPENDING ON A PARAMETER}

Let $F$ be a mapping from $R^{n} \times R^{s}$ to $R^{n}$ satisfying certain conditions. In this paper some questions related to the solvability of the equation $F(x, a)=0$, where $a$ is a parameter, are considered. An assumption is made that for a fixed value $a_{0}$ of the parameter, the equation has a solution $x\left(a_{0}\right)$. It is proved that if any other value $a$ of the parameter is sufficiently close to $a_{0}$, then the equation has a solution $x(a)$. The distance between $x(a)$ and $x\left(a_{0}\right)$ is estimated.

Basing on these results, the equation depending on a random parameter is considered. Probabilistic estimates for the -solvability of this equation as 'well as for its solution are found. At the end the equation with a random parameter is considered as a random equation and the existence of its random solution is established. 